Design and fabrication of an ac-electro-osmosis micropump with 3D high-aspect-ratio electrodes using only SU-8

This content has been downloaded from IOPscience. Please scroll down to see the full text. 2011 J. Micromech. Microeng. 21035018

(http://iopscience.iop.org/0960-1317/21/3/035018)

View the table of contents for this issue, or go to the journal homepage for more

Download details:

IP Address: 152.78.66.59

This content was downloaded on 01/12/2014 at 12:05

Please note that terms and conditions apply. 


\title{
Design and fabrication of an ac-electro-osmosis micropump with 3D high-aspect-ratio electrodes using only SU-8
}

\author{
Hamza A Rouabah ${ }^{1}$, Benjamin Y Park ${ }^{2}$, Rabih B Zaouk ${ }^{2}$, \\ Hywel Morgan ${ }^{1}$, Marc J Madou ${ }^{2}$ and Nicolas G Green ${ }^{1}$ \\ ${ }^{1}$ School of Electronics and Computer Science, University of Southampton, Southampton, UK \\ ${ }^{2}$ Mechanical and Aerospace Engineering Department, University of California, Irvine, CA, USA \\ E-mail: har04r@ecs.ac.uk
}

Received 2 September 2010, in final form 16 December 2010

Published 17 February 2011

Online at stacks.iop.org/JMM/21/035018

\begin{abstract}
Lab-on-a-chip devices require integrated pumping and fluid control in microchannels. A recently developed mechanism that can produce fluid flow is an integrated ac-electro-osmosis micropump. However, like most electrokinetic pumps, ac-electro-osmotic pumps are incapable of handling backpressure as the pumping force mechanism acts on the surface of the fluid rather than the bulk. This paper presents a novel 3D electrode structure designed to overcome this limitation. The electrodes are fabricated using carbon-MEMS technology based on the pyrolysis of the photo-patternable polymer SU-8. The novel ac-electro-osmosis micropump shows an increase in the flow velocity compared to planar electrodes.

(Some figures in this article are in colour only in the electronic version)
\end{abstract}

\section{Introduction}

Lab-on-a-chip devices have the potential to revolutionize the fields of chemical and biological analysis and separation sciences. Research and development in this area have increased rapidly over the last decade, with an emphasis on applications that require pumping of fluid, often particle laden in microchannels [1, 2]. Much effort has been exerted to fabricate mechanical micropumps, such as check-valve micropumps, peristaltic micropumps, valve-less rectification micropumps, and rotary micropumps [3].

These novel mechanical micropumps all utilize moving components to move fluids, and the long-term running of these moving parts remains a serious concern for the life time of the micropump. Often, mechanical micropumps involve mechanical actuators which translate electrical energy into mechanical work; thus, the size of the overall micropump is restricted to the size of that actuator, making them difficult to downscale. In addition, most mechanical micropumps require significant electrical energy for them to function, accompanied by significant thermal losses. Mechanical micropumps also typically feature complex multilayer structures, with many fabrication steps, and several different materials, thus increasing the fabrication time and cost of the device.

In contrast, non-mechanical micropumps have no moving parts, presenting an important advantage for long-term reliability. One example of such non-mechanical pumps is the ac-electro-osmosis (ACEO) micropump, which uses electric fields generated by a set of arrayed electrodes to induce electrokinetic pumping [4-8]. The latter micropump offers ease of fabrication, low driving potentials, and the simplicity of downscaling and integrating the pump with other components. It also has an advantage over dc-electro-osmotic pumps in that the driving force can be localized where required. The velocity magnitude with which ACEO pumps can deliver accurate steady fluid flows depends on the applied ac potential and frequency, fluid conductivity, and the electrode sizes and geometries [9]. Some effort has been devoted to the optimization of the maximum flow rate that these pumps can deliver $[10,11]$. ACEO pumps fluid using a surface force that arises from the interaction between the electrical double layer (EDL) at the surface of the electrodes and the electric field 


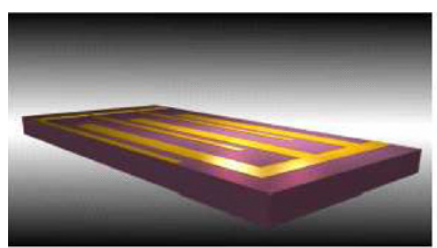

(a)

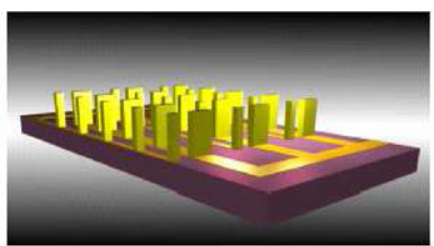

(b)

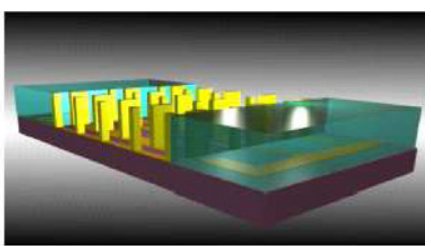

(c)

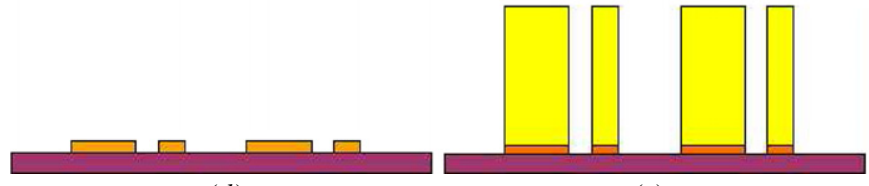

$(d)$ (e)

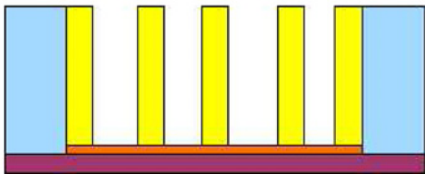

(f)

Figure 1. A schematic illustrating the evolution from planar electrodes to the new $3 \mathrm{D}$ electrode configuration. A $3 \mathrm{D}$ representation is shown in the top part and a cross-section view is shown in the bottom part. (a, d) Asymmetric planar electrodes patterned on top of a silicon wafer. (b, e) 3D high-aspect-ratio (HAR) electrodes are built on the top of the planar electrodes and (c, d) the 3D electrodes are placed within a fluidic channel.

generated by the applied ac signal [5, 6]. It is a second-order effect, as both sign of most of the charge in the double layer and the direction of the applied field change in each half cycle of the applied ac signal. The force in the fluid is generated entirely within the EDL, which, depending on the conductivity of the fluid, is only 1-30 nm in thickness compared to typical channel dimensions of 5-500 $\mu \mathrm{m}$. As a result, when a significant back pressure is applied to the pump, there is little hydrodynamic resistance to the fluid recirculating in the channel above the electrodes and therefore producing no net flow external to the micropump. Consequently, a good pump design should have as high an internal resistance as possible to ensure that the external flow rate is maintained against as high a back pressure as possible.

In the case of an ACEO pump, the internal resistance can be increased, for example, by increasing the length of the pump as the hydrodynamic resistance depends linearly on length. However, an alternative has been demonstrated in electroosmotic pumps which use frits to create porous structures with small internal channels and simultaneously large surface areas and therefore large areas of double layer [32]. A significant improvement in pump performance is achieved both because the same volume of fluid is being moved by a much larger surface drag force and, as the hydrodynamic resistance of a pipe is inversely proportional to radius to the fourth power, the internal resistance of the whole pump is greatly increased.

This can be achieved in an ACEO pump by dividing the channel cross-section into multiple channels in parallel which, therefore, have a significantly higher resistance than a single channel of the same cross-section. Our proposed design is based on extending existing asymmetric electrode arrays vertically by incorporating high-aspect-ratio (HAR) pillar electrodes dividing up the channel. More driving surface is produced for the same volume and the internal hydrodynamic resistance is increased, leading to greater pumping efficiencies. Based on these criteria, the design uses HAR asymmetric pillar electrodes placed inside a microfluidic channel (see figure 1).

Fabricating HAR ( $>5: 1)$ electrodes is a challenge in most standard fabrication methods. It is difficult to make
HAR moulds with sufficient resolution for electroplating, particularly with dimensions of less than $20 \mu \mathrm{m}$. Moulds with these dimensions can be made from SU-8 but the mould is difficult to remove afterwards. It is also difficult to control the plating rate for a dense array of electrodes with a set of different sizes. Lastly, this type of processing is expensive and time consuming. Although using conductive polymer such as conductive SU-8 $[12,13]$ to make 3D electrodes' structures represents a plausible solution, the thickness and resolution remain a problem in addition to the difficulty of making small structures $(<20 \mu \mathrm{m})$.

In this work, HAR structures are fabricated using Carbon-MEMS technology (C-MEMS), where polymer microstructures are pyrolyzed at high temperature with forming gas to produce conductive carbon structures $[14,15]$. The application of C-MEMS to dielectrophoresis has been demonstrated previously [16-19]. The structures are biocompatible, chemically inert and easy to fabricate. This paper presents details of the fabrication process of the micropump emphasizing together with a discussion of fabrication issues. Good repeatability of the structures before and after the pyrolysis step is demonstrated. The device is packaged within an SU-8 microfluidic channel, producing an electrically active device made from pyrolyzed SU-8 and a fluidic confinement structure. Preliminary tests demonstrate the functionality of the completed device and a comparison between this device and a planar asymmetric pump is presented.

\section{Fabrication}

SU-8 photoresist was processed with an MA6 Karl Suss mask aligner with a broadband UV source. The humidity in the cleanroom was controlled to be between $45 \%$ and $55 \% \mathrm{RH}$. Silicon substrates were used (4 inch diameter Si wafers with $5000 \AA \mathrm{SiO}_{2}$ thermally deposited) due to the requirement for a high pyrolysis temperature $\left(900{ }^{\circ} \mathrm{C}\right)$. The oxide layer is required to electrically isolate the electrodes and contacts from the underlying silicon. Since the designed dimensions of the small pillars are $10 \times 10$ and $20 \times 20 \mu \mathrm{m}^{2}$ features, 


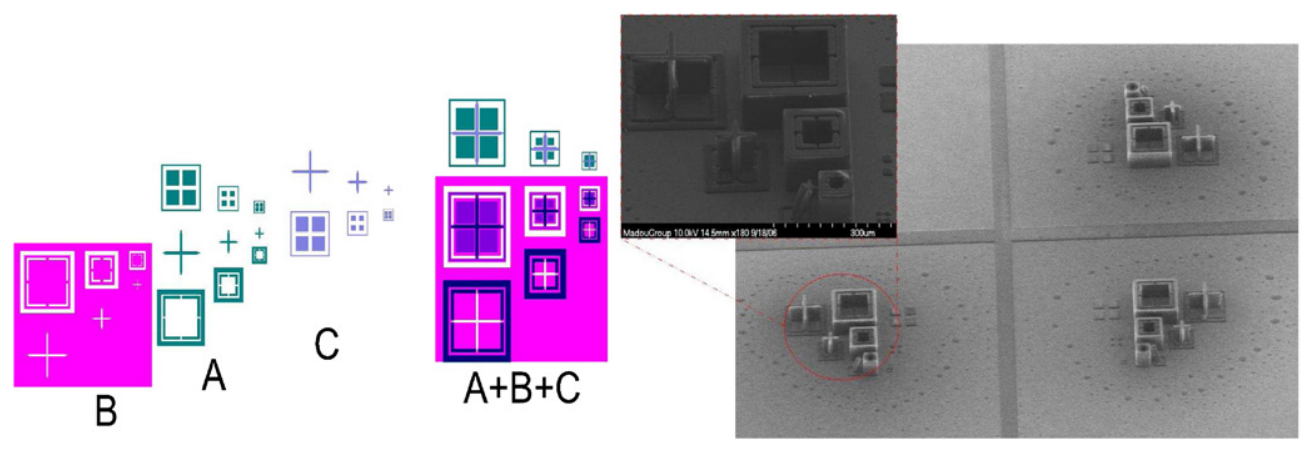

(a) Alignmant marks layouts of the 3 layers

(b) Planar and 3D-HAR alignment marks

Figure 2. (a) The alignment marks for the three layers (A, B, and C), shown separately and overlaid, consist of crosses inset in squares. (b) SEM images of the printed alignment marks of the planar and 3D pillar electrode layers made from SU-8.

for good resolution, high quality chromium photomasks were used with a resolution of $0.25 \mu \mathrm{m}$. The final device design requires three masks for (I) the base electrodes, which have a dual function as both drive electrodes and connectors, (II) pillar electrode structures and (III) the microfluidic channel. However, these masks require alignment marks as a multilayer process is involved.

To align two layers on top of each other unusally is a simple process; however, in order to align a third layer on top of two thick layers is a challenging process as the vision of the alignment marks through the microscope becomes blurry in some cases. Therefore to overcome this problem, two different alignment points with two different thicknesses (planar and 3D HAR) were used to align the third layer accurately. The alignment layouts for the three layers (A, B, and C) are shown in figure 2(a), where figure 2(b) shows the thicknesses of alignment marks. The ' + ' sign was used as the baseline for the alignment marks design as it helps to align in horizontal and vertical directions. For good alignment precision, the smallest features and gap dimensions of the alignment marks were designed to have the same size as the smallest features of the design (which is $10 \mu \mathrm{m}$ ).

In this paper, two different classes of devices were made: devices with planar electrodes only, and devices with both planar and 3D HAR electrodes. The fabrication steps were as follows.

At first, the planar and 3D HAR electrodes were optimized and combined, and then the pyrolysis step was performed followed by electrical testing. Finally, the channels were fabricated and the devices were packaged. This section details the steps and issues encountered during the fabrication process with the last sub-section summarizing the final fabrication process of the complete device.

\subsection{Planar electrodes and connections}

In order to reduce the cost of the fabrication and reduce the number of masks, the external electrical pads, the internal connectors and the planar electrodes inside the channel were drawn on the same mask. The optimum thickness for this layer was chosen to be $5 \mu \mathrm{m}$ before pyrolysis.

A $5 \mu \mathrm{m}$ layer of SU-8 5 was patterned using the parameters recommended by the SU-8 supplier Microchem
[20]. Pyrolyzation was performed for $1 \mathrm{~h}$ at $900{ }^{\circ} \mathrm{C}$ in $95 \% \mathrm{~N}_{2}$ and $5 \% \mathrm{H}_{2}$ forming gas, as described in [14]. The resulting structure is an asymmetric carbon electrode pattern with a thickness of approximately $4 \mu \mathrm{m}$. Figure 3 shows that the patterned planar electrodes are not flat, but curved instead due to shrinkage, which occurs during the pyrolysis process [21]. The shrinkage is due to the evaporation of the remaining developer, loss of water molecules, hydrogen molecules and all other species that can volatize at $900{ }^{\circ} \mathrm{C}$, except for carbon, which crosslinks with itself forming the glassy carbon electrodes. The curved surface makes the fabrication of HAR pillars on top of the bumped surface in the next step a challenge. The intention was to first fabricate the planar electrodes layer, perform pyrolysis, and test the electrical conductivity before progressing to the next step. Instead it was found that the pillar layer has to be patterned on top of the flat planar SU-8 layer prior to pyrolysis of both layers at the same time.

\subsection{D HAR electrodes}

The HAR conductive pillars were fabricated by patterning a thick SU-8 layer on top of the planar electrodes and converting both to carbon together. Due to the shrinkage effect during pyrolysis, the SU-8 structure reduces by up to $75 \%$. Therefore, the patterned polymer structures need to be 3 to 4 times the final height after pyrolysis. Since the desired height of the final carbon electrodes is around $50 \mu \mathrm{m}$, the spun-on SU-8 layer has to be around $150 \mu \mathrm{m}$. The electrodes' set is a dense array with gaps of $10 \mu \mathrm{m}$ and $20 \mu \mathrm{m}$ and with aspect ratios greater than $7: 1$. The resulting pillars should be squares and rectangles with widths of less than $5 \mu \mathrm{m}$.

2.2.1. Drag effect. Since the thickness of the HAR electrodes has to be at least $150 \mu \mathrm{m}$, a thick photoresist such as SU-8 100 is required. However, due to the high viscosity of SU-8 $100(51500 \mathrm{cSt})$, upon the spinning process due to generated viscous and centrifugal forces, the thin patterned layer of SU-8 5 (the planar electrodes on the bottom) was dragged and moved from its initial position, as depicted in figure 4. If the planar electrodes are bent, this will affect the distribution of the electric field in the final application. Therefore, in order to circumvent this problem, the viscosity 

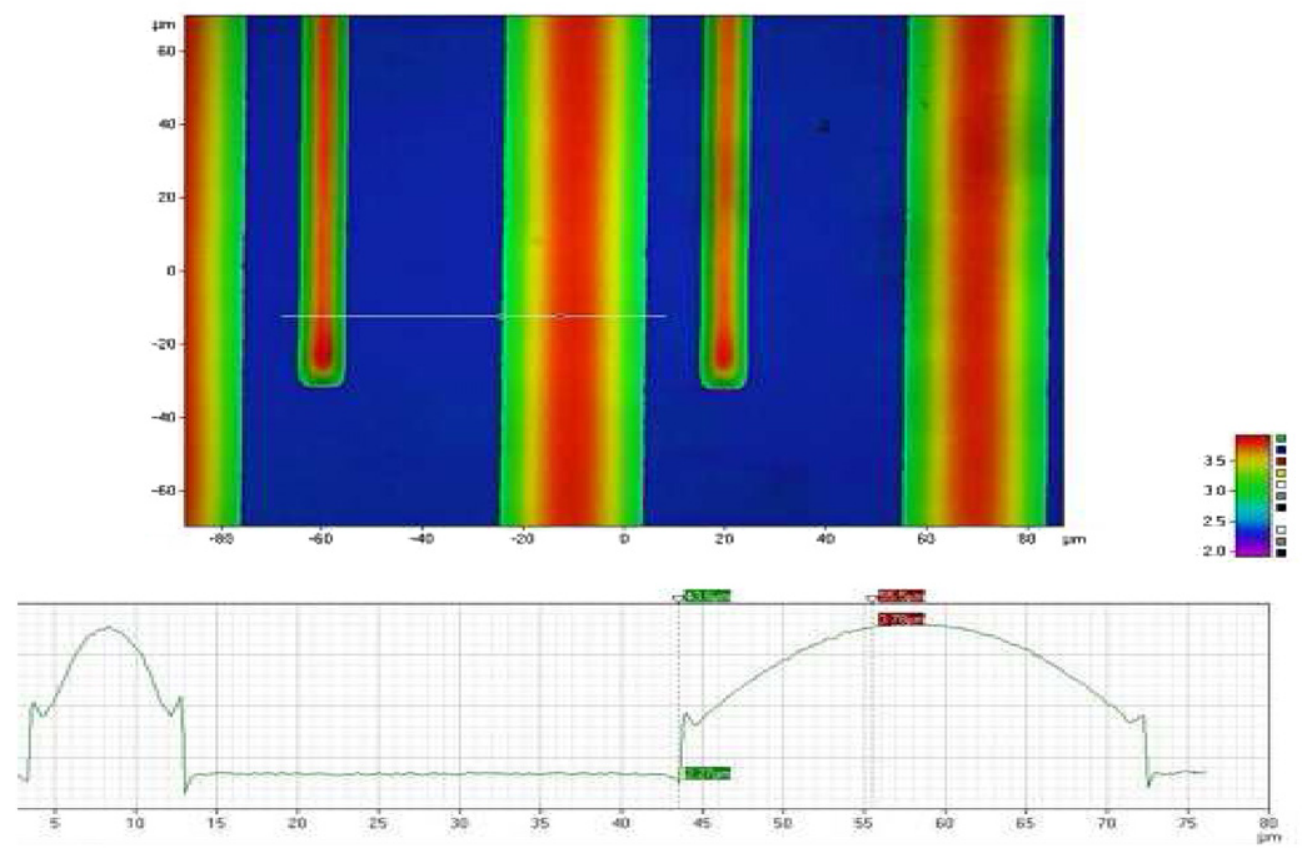

Figure 3. The profile of planar electrodes was measured with a confocal microscope after the pyrolysis process. The surface of the electrodes was changed due to shrinkage.

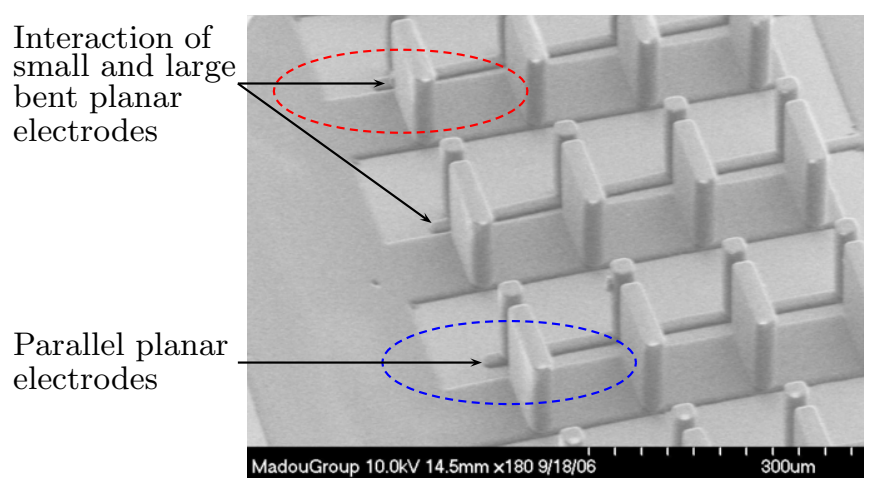

Figure 4. SEM image showing SU-8 asymmetric pillars patterned on top of planar electrodes before the pyrolysis process. The circled areas show the drag effect, where the planar electrodes were dragged by the highly viscous top photoresist during the second spinning step in the photolithography process. It can be clearly seen that some of the thin electrodes and large electrodes are in contact in the red circled area; by comparison, the lower (blue) circled area shows an undisturbed pair of electrodes.

of the SU-8 100 was reduced using the organic solvent gammabutyrolactone (GBL), or by using multiple coats of SU-8 50 , where its viscosity is lower than SU-8 100 (12250 cSt).

2.2.2. T-topping and gap elimination. The most extreme forms of HAR SU-8 structures were fabricated using the LIGA process, which relies on a monochromatic x-ray source [22]. However, using a broadband UV source, the process for making the HAR structure becomes more challenging [23, 24], especially for a dense configuration with narrow gaps between the structures.
In this work, the process was optimized to give clear gaps with vertical resist side walls with a best aspect ratio below 5:1. For thicker layers, the absorbance of the SU- 8 and the variation in exposure dose with depth results in several problems. With an exposure dose calculated for the whole thickness of the resist used, insufficient cross-linking occurs at the base of the pillars. Figure 5(a) shows a typical result. The pillars have collapsed against each other due to the lack of structural rigidity, close to the substrate. A small increase in the exposure dose leads to the appearance of the so-called T-topping, which refers to a negative shaped sidewall profile, as shown in figure $5(b)$. The underlying reason is that the top part of the resist is exposed more to the UV light than the bottom part, despite the fact that the full SU-8 film is exposed for the same period of time: the absorbance of the UV-light by the SU-8 layer from the top to the bottom decreases gradually.

Increasing the UV dose further does not correct the problem, pillars are still structurally weak at the base, but now the upper part of the pillars is severely over exposed and the smaller gaps do not develop, as shown in figure 5(c).

This problem was found to be less related to the exposure dose but rather to the spread of UV wavelengths generated. The shorter wavelength components of UV light are absorbed by the top surface layer, while the longer wavelength components penetrate further to the bottom. Longer wavelengths (i-line, g-line and h-line) with lower absorbance are used to allow high intensity of UV light to reach to the bottom part of the SU-8 layer [25].

Therefore an SU-8 filter was used to block out wavelengths shorter than $350 \mathrm{~nm}$ [26], which was made from a $100 \mu \mathrm{m}$ thick SU-8 layer spun onto a photomask. This homemade SU-8 filter gave good results with high resolution: an aspect ratio of more than 10:1 for SU-8 structures with 


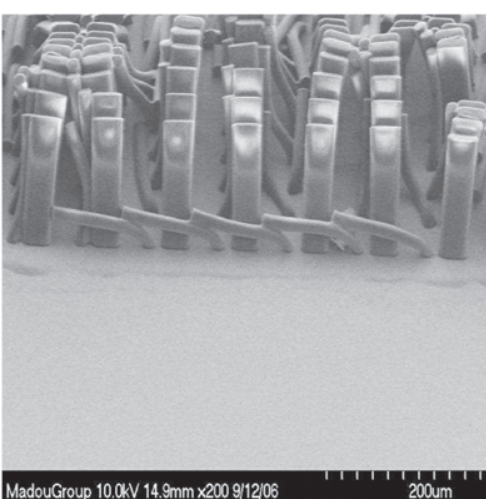

(a)

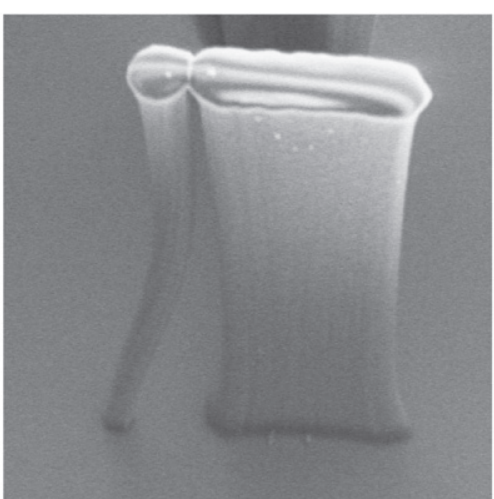

(b)

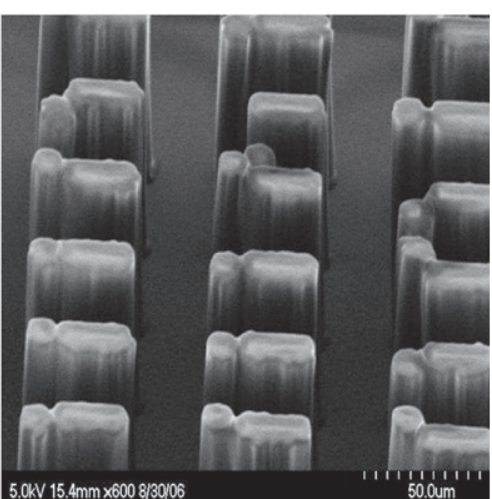

(c)

Figure 5. (a) SEM image showing HAR structures collapsed against each other due to the lack of structural rigidity, close to the substrate. (b) SEM image showing the initiation of the T-topping effect where the pillars have a mushroom shape with a negative wall shape. (c) SEM image showing the effect of increasing the exposure dose: the pillars still demonstrate a lack of rigidity and the smallest gaps cannot be developed anymore.

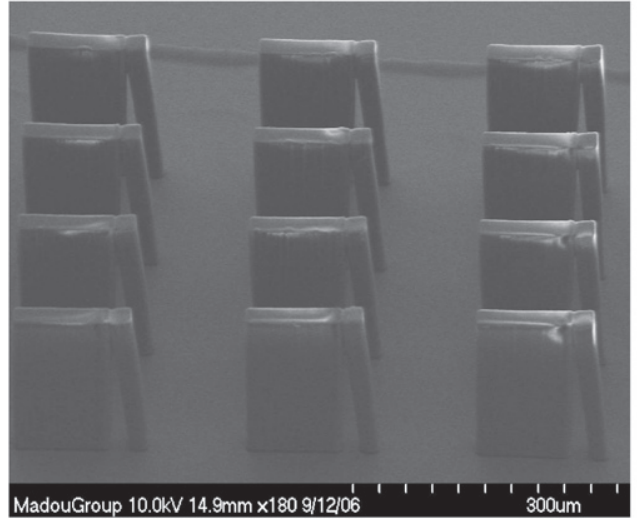

(a)

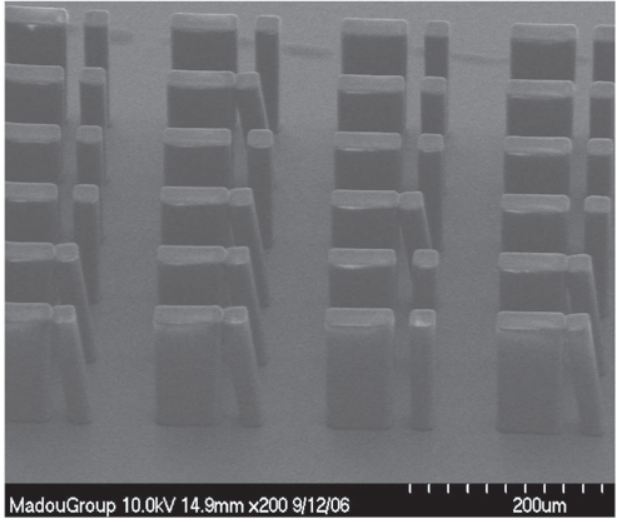

(b)

Figure 6. SEM pictures showing the stiction effect. In the left panel, stiction occurs on all of the pillars. In the right panel, a partial stiction effect occurs on a few pillars only.

$10 \mu \mathrm{m} / 30 \mu \mathrm{m}$ and $20 \mu \mathrm{m} / 60 \mu \mathrm{m}$ (length of small pillar/length of large pillar electrodes) and small gaps of $10 \mu \mathrm{m}$ and $20 \mu \mathrm{m}$.

2.2.3. Stiction effect. It was observed that the tops of post pairs were stuck together, as shown in figure 6. Initially it was thought that this was due to the aggressive agitation during the development step; however, it turned out to be a stiction effect [27].

During the development process, the developer solution rises between the walls of each pair of the pillars as a result of the capillary forces during the solvent evaporation. As this occurs, and just before droplets of the developer evaporate, it brings the two pillars' heads together because the pillars are high enough and so are very sensitive to any motion from the top. They could not withstand the capillary forces as the surface tension of the developer was high enough to bend them. The pillars' heads were then stuck firmly together.

In order to work out this problem, a dry release process can be used [28-30]. However, it requires a special setup; therefore a wet release similar to [31] was applied. After the development steps, immediately, the wafer was immersed for
$2 \mathrm{~min}$ in an isopropanol container and then for another $2 \mathrm{~min}$ in methanol to ensure that the pattern was filled with methanol to replace both SU-8 developer and isopropanol. Methanol has low surface tension compared to the developer solution and prevents stiction [31].

\subsection{Combination of planar electrodes and $3 D$ electrodes}

When the SU-8 50 layer was deposited on top of the patterned SU-8 5 layer, the alignment marks became invisible, as both photoresists have the same refractive index. To solve this problem, coloured SU-8 can be used, or the alignment marks can be covered by tape before spinning on the SU-8. The tape is then removed immediately after the softbake step to allow the alignment and UV-exposure process.

Using this technique, an array of HAR electrodes was successfully patterned on top of an asymmetric set of planar electrodes. Both layers were pyrolyzed to create conductive carbon post electrodes on top of planar electrodes and contacts. Shrinkage during the pyrolysis step is clearly seen in figure 7, where the initial dimensions of the SU-8 structure were isotropically reduced. A uniform structure was obtained with 


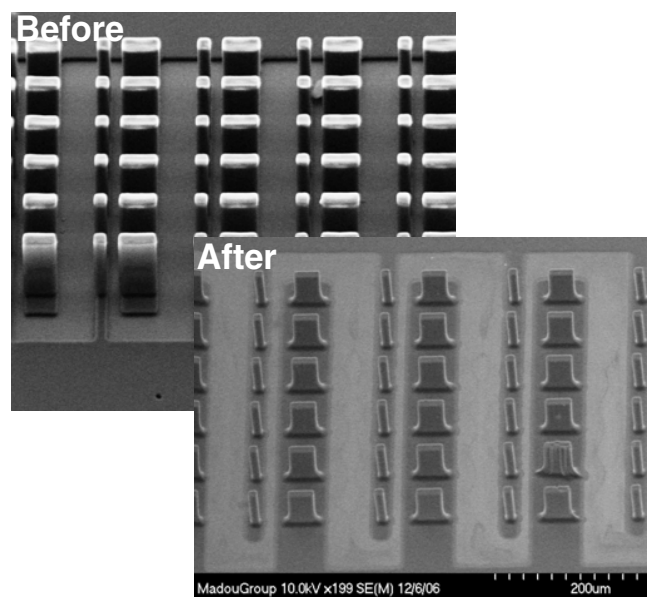

(a)

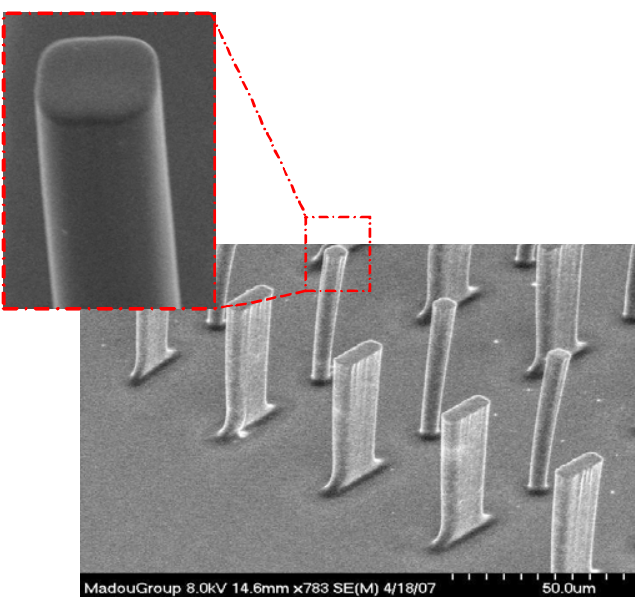

(b)

Figure 7. (a) SEM images showing the set of HAR pillars before and after pyrolization. (b) SEM image showing the HAR pyrolyzed electrode arrays (12:1) with the smallest dimensions. The dimensions of the pillars are $4 \times 4 \mu \mathrm{m}^{2}$ and $4 \times 20 \mu \mathrm{m}^{2}$ for small and large electrodes, the height is $50 \mu \mathrm{m}$ and the gap between the large and small pillars is around $20 \mu \mathrm{m}$. The high resolution and smoothness of the electrodes can be clearly seen.

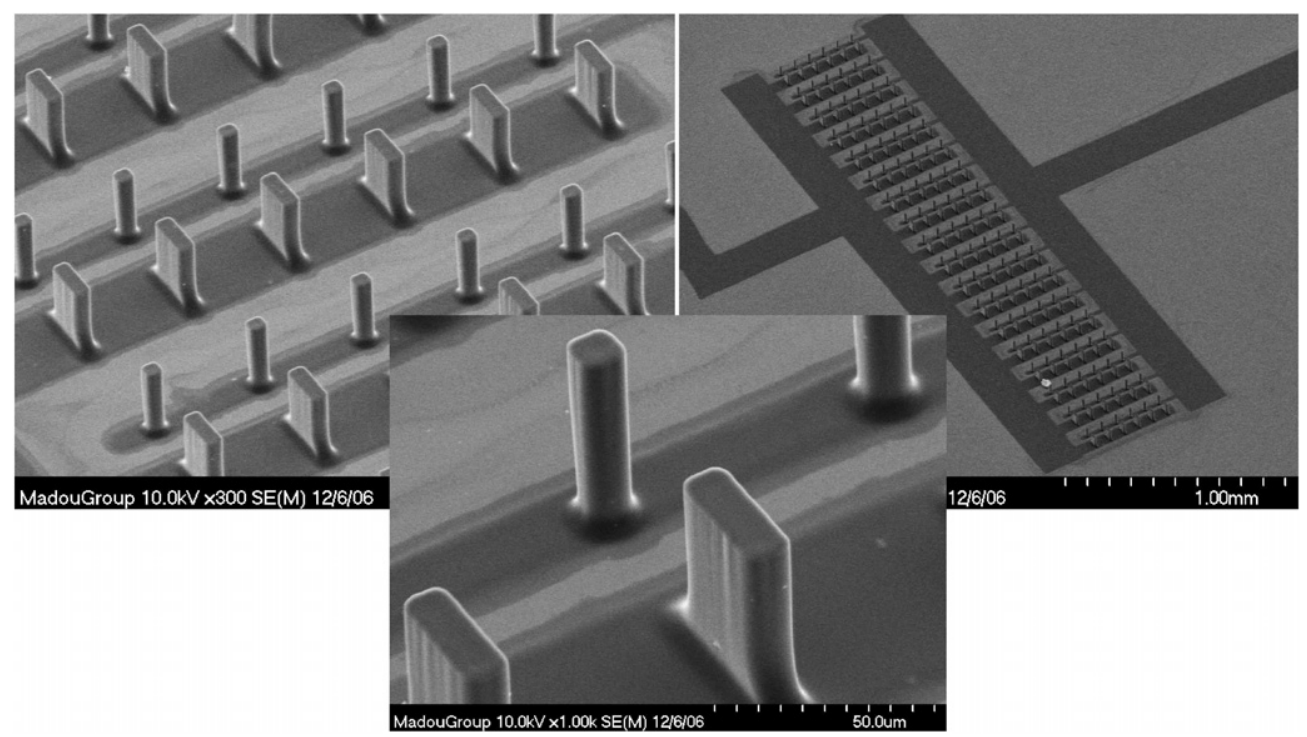

Figure 8. SEM pictures show the final 3D-electrode (HAR pillars) configuration on top of planar electrodes after pyrolysis.

smooth surfaces, as shown in figure $7(a)$. A final aspect ratio of more than 12:1 for $4 \times 4 \mu \mathrm{m}^{2}$ and $4 \times 20 \mu \mathrm{m}^{2}$ electrodes and a gap of less than $20 \mu \mathrm{m}$ were achieved (figure $7(b)$ ). The finished device is shown in figure 8 .

\subsection{Electrical inspection}

After the pyrolysis process, the electrical resistance between the wide and the narrow electrodes was measured. This should be larger than $2 \mathrm{M} \Omega$ but was found to be between $13.8 \mathrm{k} \Omega$ and $2 \mathrm{M} \Omega$, as shown by the bar chart in figure 9 . This short circuit is due to the carbon residue between the narrow and wide electrodes (see figure 9), from the pyrolysis of unavoidable remaining traces of SU-8 that were not removed.

This carbon could not be prevented by increasing the developing time or by rinsing the wafer with the SU-8 developer. This layer was absorbed by the oxide layer,

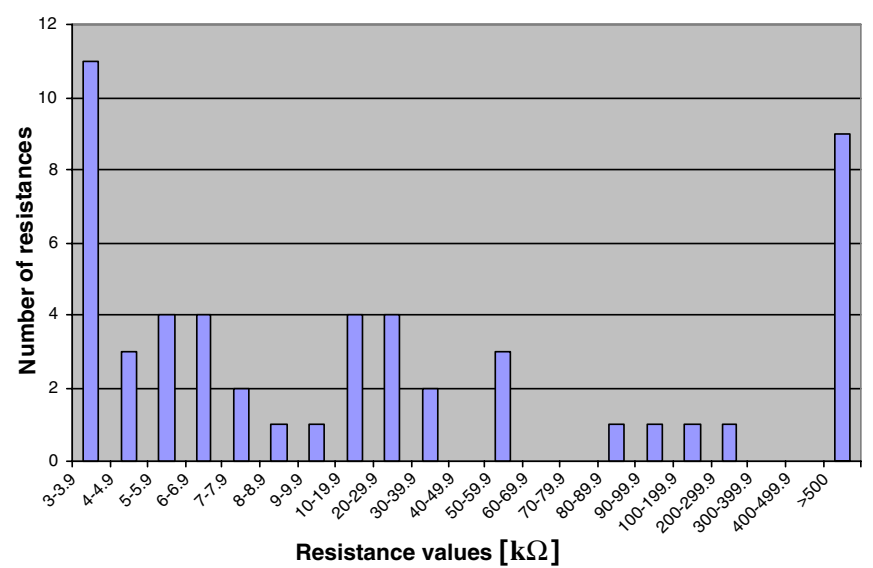

Figure 9. Bar chart illustrates the percentage of the devices resistance after pyrolysis. 


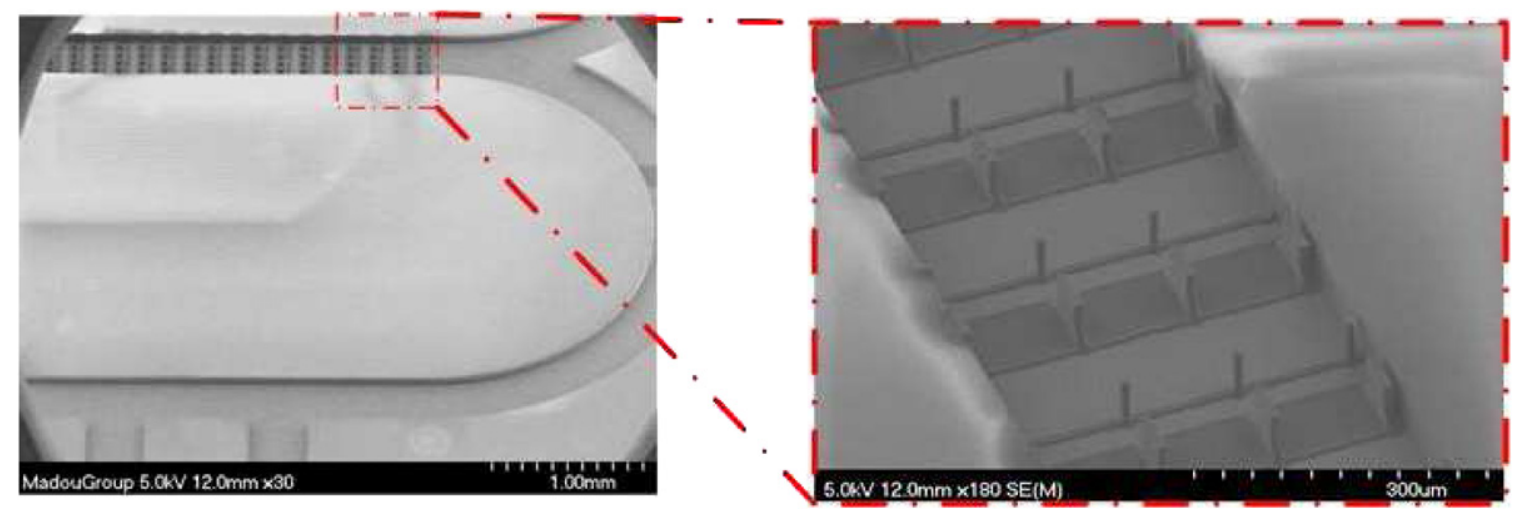

Figure 10. The final device is packaged by the fabrication of a microfluidic channel in SU8. A loop channel is constructed to allow low resistance measurements.

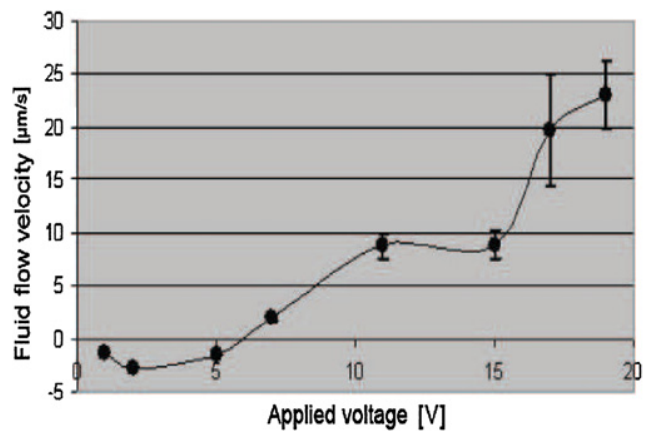

(a)

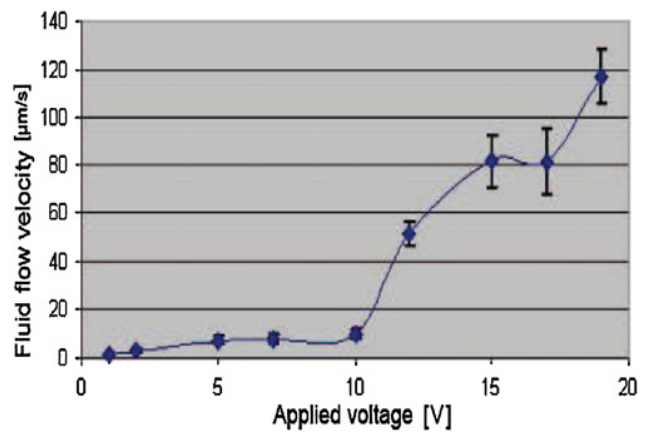

(b)

Figure 11. Fluid flow velocity for planar electrodes. (a) Fluid flow velocity for the planar electrodes. (b) Fluid flow velocity for 3D electrodes.

Table 1. Variation in the resistance between the electrodes before and after the exposure to $\mathrm{O}_{2}$ plasma etch.

\begin{tabular}{ll}
\hline Before $\mathrm{O}_{2}$ plasma etch & After $\mathrm{O}_{2}$ plasma etch \\
\hline $13.8 \mathrm{k} \Omega$ & $29.8 \mathrm{k} \Omega$ \\
$55.3 \mathrm{k} \Omega$ & $83.8 \mathrm{k} \Omega$ \\
$87.6 \mathrm{k} \Omega$ & $173.1 \mathrm{k} \Omega$ \\
$227 \mathrm{k} \Omega$ & $2.2 \mathrm{M} \Omega$ \\
$511 \mathrm{k} \Omega$ & $2.2 \mathrm{M} \Omega$ \\
$1.1 \mathrm{M} \Omega$ & $2.2 \mathrm{M} \Omega$ \\
$2 \mathrm{M} \Omega$ & $2.2 \mathrm{M} \Omega$ \\
\hline
\end{tabular}

which acts as a sponge. It is also possible that the carbon contaminated furnace deposits a thin layer of carbon between the electrodes during the pyrolysis process. This carbon layer was removed using a slow $\mathrm{O}_{2}$ plasma etch.

While this step is unavoidable, it has the negative effect of roughening the surface of the electrodes, which may reduce the fluid velocity. The improvement in the impedance for a wide range of resistance is shown in table 1.

\subsection{Channel fabrication}

The last stage is patterning of the microfluidic channel. An oval channel with a width of $500 \mu \mathrm{m}$, a thickness of $50 \mu \mathrm{m}$ and a length of around $7 \mathrm{~mm}$ was fabricated by patterning SU-8 25 over the top of the electrode array. The 3D HAR electrodes were robust enough to withstand this fabrication step without breaking, bending, or peeling off, as shown in figure 10 .

\section{Device testing}

For proof of the concept and in order to test the functionality of the device, a low conductivity solution of $0.1 \mathrm{mM}$ of phosphate buffered saline (PBS) from Oxoid (Unipath Ltd, Basingstoke, UK) was prepared, and then mixed with $2 \mu$ m fluorescent latex beads and injected into the channel. Two designs were used:

(1) a planar array with dimensions: gap/electrode/gap/ electrode of 60:60:20:20 $\mu \mathrm{m}$

(2) a 3D array with the same dimensions of gaps and electrodes, with $50 \mu \mathrm{m}$ high pillars spaced at $74 \mu \mathrm{m}$ along the electrode (across the channel).

At a fixed frequency of $1 \mathrm{kHz}$ and a voltage varying between $1 V_{p p}$ and $19 V_{p p}$, preliminarily results indicate that this C-MEMS ACEO pump technology is able to generate fluid flow.

For the 2D device, a slight reverse pumping was noticed at low voltages in contrast to the $3 \mathrm{D}$ device. A maximum fluid flow velocity of $22.6 \mu \mathrm{m} \mathrm{s}^{-1}$ was achieved at $19 V_{p p}$ for the planar electrode configuration (see figure 11(a)). However, the 3D micropump had a velocity five times higher of approximately $120 \mu \mathrm{m} \mathrm{s}^{-1}$ at $19 V_{p p}$ for the same frequency 
of $1 \mathrm{kHz}$ (see figure $11(b)$ ). This increase in the fluid flow velocity was due the increase in the surface of the electrodes, which in turn increases the EDL surface and hence increases the ACEO pumping. Additional improvement of the fluid velocity would be achievable by optimizing the dimensions of the electrodes.

\section{Conclusion}

In this paper, C-MEMS technology was used to construct 3D high-aspect-ratio pillar electrodes for a novel ac-electroosmotic micropump. The device was constructed solely from SU-8 photoresist, including the connectors, planar electrodes, the HAR pillars, and the channel using the standard UVphotolithography process. Finally, tests indicate that firstly C-MEMS structures are capable of generating fluid flow. Secondly, the device with HAR electrodes pumped fluid with a velocity five times greater than a similar 2D design.

\section{Acknowledgments}

The authors would like to thank Jake Hes, Vu Phan, David Crosely, Genis Teixidor, Dr Mo Kebaili, and Dr A Boufarouk for their technical support.

\section{References}

[1] Morgan H, Green N, Ramos A and Garcia-Sanchez P 2007 Control of two-phase flow in a microfluidic system using ac electric fields Appl. Phy. Lett. 91251407

[2] Holmes D, Morgan H and Green N G 2006 High throughput particle analysis: combining dielectrophoretic particle with confocal optical detection J. Biosens. Bioelectron. 21 1621-30

[3] Nguyen N T, Huang X and Chuan T K 2002 MEMS-micropumps: a review J. Fluids Eng. 124 384-92

[4] Wong P K, Wang T H, Deval J H and Ho C M 2004 Electrokinetics in micro devices for biotechnology applications IEEE/ASME Trans. Mechatronics 9 366-76

[5] Green N G, Ramos A, Gonzalez A, Morgan H and Castellanos A 2000 Fluid flow induced by nonuniform ac electric fields in electrolytes on microelectrodes: I. Experimental measurements Phys. Rev. E 61 4011-18

[6] Green N G, Ramos A, Gonzalez A, Morgan H and Castellanos A 2002 Flow induced by nonuniform ac electric fields in electrolytes on microelectrodes: III. Observation of streamlines and numerical simulation Phys. Rev. E 66026305

[7] Ajdari A 2000 Pumping liquids using asymmetric electrode arrays Phys. Rev. E 61 R45-8

[8] Urbanski J P, Levitan J A, Burch D N, Thorsen T and Bazant M Z 2007 Porous glass electroosmotic pumps: design and experiments $J$. Colloid Interface Sci. $309332-41$

[9] Gonzalez A, Ramos A, Green N G, Castellanos A and Morgan H 2000 Fluid flow induced by nonuniform ac electric fields in electrolytes on microelectrodes: II. A linear double-layer analysis Phys. Rev. E 61 4019-28

[10] Ramos A, Gonzalez A, Castellanos A, Green N G and Morgan H 2003 Pumping of liquids with ac voltages applied to asymmetric pairs of microelectrodes Phys. Rev. E 67056302
[11] Bazant M Z and Ben Y 2006 Theoretical prediction of fast 3D AC electro-osmotic pumps Lab on a Chip 6 1455-61

[12] Jiguet S, Bertsch A, Hofmann H and Renaud P 2004 Conductive SU8 photoresist for microfabrication J. Adv. Eng. Mater. 6 719-24

[13] Jiguet S, Bertsch A, Judelewicz M, Hofmann H and Renaud P 2006 SU-8 nanocomposite photoresist with low stress properties for microfabrication applications J. Microelectron. Eng. 83 1966-70

[14] Chunlei W, Taherabadi L, Guangyao J, Madou M J, Yeh Y and Dunn B 2004 C-MEMS for the manufacture of 3D microbatteries Electrochem. Solid-State Lett. 7 A435-8

[15] Chunlei W, Guangyao J, Taherabadi L H and Madou M J 2005 A novel method for the fabrication of high-aspect ratio C-MEMS structures J. Microelectromech. Syst. $14348-58$

[16] Park B Y, Paradiso A, Kawabe M and Madou M J 2006 Novel dielectrophoretic filtration methods and designs Proc. SPIE 6172 61720C1-10

[17] Martinez-Duarte R, Rouabah H A, Green N G, Madou M J and Morgan H 2007 Higher efficiency and throughput in particle separation with 3D dielectrophoresis with C-MEMS Proc. 11th Int. Conf. on Miniaturized System for Chemistry and Life Sciences: microTAS (Paris) p 826

[18] Rouabah H A, Park B Y, Zaouk R B, Morgan H, Madou M J and Green N G 2007 Fabrication of fully functional ac-electroosmosis micropump with $3 \mathrm{D}$ high aspect ratio electrodes using only SU-8 p 317 Proc. 18th MicroMechanics Europe Workshop (Guimaraes)

[19] Rouabah H A, Park B Y, Zaouk R B, Madou M J and Green N G 2008 Producing fluid flow using 3D carbon electrodes J. Phys.: Conf. Ser. 142012072

[20] http://www.microchem.com/

[21] Taherabadi L, Wang C, Zoval J and Madou M J 2005 Electrical properties and shrinkage of carbonized photoresist films and the implications for carbon microelectromechanical systems devices in conductive media J. Electrochem. Soc. 152 J136-43

[22] Vora K D, Shew B Y, Harvey E C, Hayes J P and Peele A G 2005 Process optimisation for compact, high aspect ratio SU-8 microstructures using x-ray lithography Proc. SPIE-Device and Process Technologies for Microelectronics, MEMS, and Photonics IV p 603701

[23] Williams J D and Wang W 2004 Using megasonic development of SU-8 to yield ultra-high aspect ratio microstructures with UV lithography J. Microsyst. Technol. 10 694-8

[24] Reznikova E F, Mohr J and Hein H 2005 Deep photo-lithography characterization of SU-8 resist layers $J$. Microsyst. Technol. 11 282-91

[25] Yang R and Wang W 2005 A numerical and experimental study on gap compensation and wavelength selection in UV-lithography of ultra-high aspect ratio SU-8 microstructures Sensors Actuators B 110 279-88

[26] Vlachopoulou M E, Tserepi A, Vourdas N, Gogolides E and Misiakos K 2005 2nd Conf. on Microelectronics, Microsystems and Nanotechnology p 293

[27] Wu D, Fang N, Sun C and Zhang X 2005 Stiction problems in releasing of 3D microstructures and its solution Sensors Actuators A 128 109-15

[28] Kim C J, Kim J Y and Sridharan B 1998 Comparative evaluation of drying techniques for surface micromachining Sensors Actuator A 64 17-26

[29] Haefliger D, Nordstro M, Rasmussen P A and Boisen A 2005 Dry release of all-polymer structures J. Microelectron. Eng. 78 88-92 
[30] Vora K D, Peele A G, Shew B Y, Harvey E C and Hayes J P 2007 Fabrication of support structures to prevent SU-8 stiction in high aspect ratio structures Microsyst. Technol. 13 487-93

[31] Shirtcliffe N J, Aqil S, Evans C, McHale G, Newton M I, Perry C C and Roach P 2004 The use of high aspect ratio photoresist (SU-8) for super-hydrophobic pattern prototyping J. Micromech. Microeng. 14 1384-9 [32] Yaoa S, Hertzoga D E, Zenga S, Mikkelsen Jr J C and Santiago J G 2003 The effect of step height on the performance of three-dimensional ac electro-osmotic microfluidic pumps J. Colloid Interface Sci. 268 143-53 